\title{
The Magnetic Geometry of Pulsed Astrophysical Jets
}

\author{
T. A. Gardiner, A. Frank \\ Dept. of Physics and Astronomy, \\ University of Rochester, Rochester, NY 14627-0171
}

\begin{abstract}
Hypersonic, highly collimated, mass outflows (jets) are a ubiquitous phenomena in astrophysics. While the character of the jets differ, many exhibit some form of quasi-periodic clumping indicating the jet source is episodic or pulsed. The presence of pulsed jets in so many astrophysical contexts suggests a common formation mechanism. Such a process seems to have been found in Magneto-centrifugal launching, the combination of magnetic and centrifugal forces that occurs when a magnetized gaseous accretion disk orbits a central gravitating source. Observations of strong magnetic fields in jets are, however, rare or indirect. Thus the presence and effects of magnetic fields in YSO jets remains an unresolved issue of the highest importance. In this letter we focus on what should be expected of the structure of the fields in pulsed YSO jets. We show that combining velocity variability with an initial field configuration consistent with collimated, Magneto-centrifugally launched jets leads to a clear set of predictions concerning the geometry and relative strength of the magnetic field components in evolving YSO (and perhaps other) jets.
\end{abstract}

Subject headings: ISM: jets and outflows - magnetic fields - magnetohydrodynamics: MHD

\section{INTRODUCTION}

Hypersonic, highly collimated, mass outflows (jets) are a ubiquitous phenomena in astrophysics. They occur in environments as diverse as newly forming stars, (Reipurth 1997) highly evolved stars, (Soker \& Livio 1994) neutron stars and stellar mass black holes, (Spruit 2000) and massive black holes associated with the centers of galaxies (Leahy 1991).

Accretion disks are believed to play a key role in the physics of jet launching. Infalling, rotating matter is stored in these disks until dissipation allows material to spiral inward and feed 
the central, gravitating object. Both YSO and AGN disks are believed to support strong, well ordered magnetic fields. The current consensus holds that the these fields are the agents for producing jets in a process known as Magneto-centrifugal launching. In this mechanism, plasma in the disk is loaded on to co-rotating field lines. If conditions such as the inclination angle of the field are favorable and a sufficient amount of energy is available, the plasma is centrifugally flung outward along the field lines. As the field is dragged backwards by the inertia of the plasma, strong toroidal field components are generated which collimate the outflow into a narrow jet. We note however that the external medium might also help focus the outflow. This process has been studied in detail by many authors both analytically (Heyvaerts \& Norman 1989, Pudritz 1991, Shu et al. 1994, Lery et al.1999) and through numerical simulations (Ouyed \& Pudritz 1997, Romanova et al.1998, Kudoh et al.1998)

Our goal in this letter is to show the effect of periodic velocity pulsations on the field geometry in the jet. In our studies of the effect of magnetic fields on jet propagation (Frank $e$ al. 1998, Frank et al. 2000, Gardiner et al. 1999) we have found a simple, universal relationship between velocity variability at the jet source (the "nozzle") and the evolution of the jet's magnetic field when the initial field is helical as would be expected from Magneto-centrifugal launching models. In section 2 we present analytical calculations showing the form of the evolved geometry. In section 3 we present numerical simulations which confirm the analytical models.

\section{Analytical Model}

We begin assuming that the jet can be modeled using the equations of ideal magnetohydrodynamics in cylindrical symmetry. Consider a system of cylindrical coordinates $(r, \phi, z)$ with the $z$-axis parallel to the outflow and an accretion disk in the $z=0$ plane. In magneto-centrifugal launching models for the production of astrophysical jets, a magnetic field is anchored to a differentially rotating accretion disk. Plasma loaded on to the field lines is "flung" off the disk forming a wind (Pudritz \& Konigl 2000). Differential rotation and plasma inertia then act to enhance the development of a toroidal field component $\left(B_{\phi}\right)$ whose pinch forces eventually collimate the wind into a narrow jet. Thus the general geometry for the magnetic field in a steady, cylindrically symmetric, magneto-centrifugally launched and collimated jet is that of a helix, $\mathbf{B}=\left(0, B_{\phi}(r), B_{z}(r)\right)($ Heyvaerts \& Norman 1989, Lery et al. 1998).

The field is frozen-in to the beam as it propagates. To clarify how pulsing will modify such helical field configuration, consider first the induction equation. In steady flow the poloidal components of the velocity and magnetic field are parallel. Thus for collimated flows, the column of plasma which makes up the jet will have no radial components of velocity or magnetic field $\left(B_{r}=v_{r}=0\right)$. Assuming this remains approximately unchanged during pulse propagation, the induction equation takes the form,

$$
\frac{\partial B_{\phi}}{\partial t}+\frac{\partial}{\partial z}\left(v_{z} B_{\phi}-v_{\phi} B_{z}\right)=0
$$


While $B_{\phi}$ and $B_{z}$ may be comparable (depending on initial conditions in the disk and the exact nature of the launching mechanism), the rotation rate in a jet will always be much smaller than propagation speed $\left(v_{z} \gg v_{\phi}\right)$. Equation 1 is thereby approximated by a simple conservation law with the same form as the continuity equation,

$$
\frac{\partial \rho}{\partial t}+\frac{\partial}{\partial z}\left(\rho v_{z}\right)=0
$$

Thus, in a reference frame moving with the plasma, the ratio of the toroidal field strength $B_{\phi}$ to the matter density $\rho$ is approximately independent of time.

We now consider the effect of pulsing on the jet beam dynamics. Assuming the jet beam remains approximately in equilibrium in the radial direction it is possible to develop a simple 1-D kinematic model for a jet with variable outflow speed (Gardiner et al. 1999, Smith et al. 1997, Raga et al. 1992). The essential details of this analysis are captured in Burgers' equation. When a flow exiting the nozzle of a jet varies with time, the variations propagate downstream and are modified by compression and rarefaction effects. Consider material ejected at the jet nozzle at some time $t_{\circ}$ with velocity $v_{j}\left(t_{\circ}\right)$. If $v_{j}^{\prime}\left(t_{\circ}\right)>0$ (where the prime denotes a time derivative) then material ejected at $t_{\circ}$ is moving faster than material ejected earlier. The fast gas parcels encroach upon slower moving material creating regions of compression. If $v_{j}^{\prime}\left(t_{\circ}\right)<0$ then material ejected at $t_{\circ}$ is moving slower than material ahead of it. Gas parcels will move apart creating rarefaction regions of low density. If the velocity variations are strong enough compressive regions will eventually steepen to form pairs of shock waves (each pair containing an upstream and downstream facing shock). When the variations are periodic a succession of shock pairs will propagate down the beam with each pair separated by a rarefaction region.

In regions of smooth flow, away from shocks, we may combine the continuity equation with Burgers' equation to obtain a relation for the density of a gas parcel $\rho$ at time $t$ which was launched at time $t_{\circ}$ with velocity $v_{j}\left(t_{\circ}\right)$. Combining this result with the proportionality of the density and toroidal magnetic field component we express the evolution of the field geometry as

$$
\frac{B_{\phi}}{B_{z}}=\left(\frac{B_{\phi, o}}{B_{z}}\right)\left(\frac{1}{1-\kappa\left(t-t_{\circ}\right)}\right) \text { where } \kappa=\left(\frac{v_{j}^{\prime}\left(t_{\circ}\right)}{v_{j}\left(t_{\circ}\right)}\right) .
$$

In the equation above we have used the fact that the poloidal component $B_{z}$ lies parallel to the gas motions and is independent of time. Equation 3 describes how the helical magnetic field lines are compressed or stretched respecitively by increasing or decreasing velocity variations at the nozzle. While equation 3 breaks down when compressive regions steepen into shocks, consideration of the MHD jump conditions allows the post-shock field conditions to be calculated. When post-shock cooling is included the compression of the toroidal field can become quite large $B_{\phi} / B_{\phi, o}>10$. Thus from simple dynamical arguments we infer that a pulsing jet with an embedded helical field will inevitably develop a periodic field structure consisting of rarefied regions where the helix is combed out and has a more poloidal geometry alternating with denser, toroidally dominated knots. The rarefaction regions may become poloidally dominated depending on the initial value of $\frac{B_{\phi}}{B_{z}}$ and the age of the jet (the ratio asymptotically decreases as $1 / \kappa t$ ). 


\section{Numerical Simulations}

To confirm the predictions of our simple 1-D model we have performed axisymmetric (2.5-D) numerical simulations of a pulsed radiative MHD jet. The conditions in our model are appropriate to protostellar jets with an initial number density, velocity, temperature and sonic Mach number in the jet of $120 \mathrm{~cm}^{-3}, 200 \mathrm{~km} \mathrm{~s}^{-1}, 2.5 \times 10^{3} \mathrm{~K}$ and 34 respectively. The jet was initialized with a dynamically weak helical field (plasma $\beta \approx 10 \rightarrow 10^{3}$ and $\frac{B_{\phi, o}}{B_{z, o}} \approx 0 \rightarrow 10$ ). The toroidal field strength varies with an approximately paraboloidal shape as a function of radius peaking at $1 / 2$ the jet radius. The $z$-component of the magnetic field was chosen to be uniform and permeating both the jet and ambient medium. The pressure and tension forces associated with the toroidal field in the jet are balanced by the gas pressure. The velocity at the jet nozzle was varied with a sinusoidal function $\left(v_{j}(t)=v_{o}[1+A \sin (\omega t)]\right)$ where $A=0.25$ and the pulsation period was 80 years. The jet was driven into a constant ambient medium with density and temperature 60 $\mathrm{cm}^{-3}$ and $5 \times 10^{3} \mathrm{~K}$ respectively. In Figures $1 \mathrm{a}$ and $1 \mathrm{~b}$ we show the density and magnetic field structure from the simulation. The density image shows the high density regions behind the bow shock at the head of the jet. In addition there are two high density knots in the beam where compressive regions have steepened into shocks and a third region of compression near the base of the jet. The magnetic field image shows that the intensity of the toroidal field is highest behind the shocks. In particular note the concentration of toroidal field in the knots. Note also that the $B_{z}$ component is relatively unaffected by the dynamics except at the jet edges or where radial gas motions are important, such as near the head of the jet. In Fig 2 we show a plot of $B_{\phi}(z)$ at two times in the evolution of the simulation which differ by $1 / 2$ of a pulsation period. The growth of the toroidal field component as velocity pulses steepen into shocks is clearly evident as is its rarefaction in the region between the pulses. Note that our simulation shows a maximum increase of $\frac{B_{\phi}}{B_{\phi, o}} \approx 30$. From the plot it appears as if the ratio of the maximum to the minimum toroidal field strength has stabilized at $\approx 30$. Plotting a similarly scaled density profile with the toroidal field strength shows the approximations made to be quite acceptable. In the rarefaction regions $B_{\phi}$ continues to decrease at the end of the simulation. Thus in proto-stellar jets, whose lifetimes are two orders of magnitude larger we expect the rarefaction regions to become poloidally dominated.

Thus the numerical simulations confirm the results of our simple model. In flows with strong cooling, helical fields embedded in pulsed jets will invariably lead to toroidally dominated knots separated by low density regions which can be poloidally dominated. Our model allows a clean prediction of the field structure in velocity variable jets. Polarization maps (the most promising means of determining field structure, Ray et al. 1996, Chrysostomou et al.2000, Minchin ea 1995) should show polarization vectors whose orientation angle changes by $90^{\circ}$ at the boundary of bright knots. Our model also allows one to cleave between different models of the origin of knots based on the observed field structure. There is currently some debate over the origin of knots in protostellar flows. It remains unclear if the knots are due to pulsing of the jet source or instabilities in the beam, though recent observations make a strong case for pulsing in the case of jets from young stars. Zinnecker et al. 1998 While shock waves will occur in a hypersonic beam which becomes 
unstable they will be both weak and oblique. Stone et al. 1997 Thus instabilities are not likely to lead to the strong toroidal/poloidal (compression/rarefaction) regions predicted by our model.

\section{Conclusions}

We have shown that pulsating jets with initially helical fields will evolve to an alternating poloidal/toroidal dominated field geometry. The strongest toriodal field will be confined to the dense knots in between the shock pairs while the rarefaction regions will contain a dominant polodial field.

While much of the discussion in this paper has been concerned with the geometry of the magnetic field, equally important is the magnetic field topology. It seems quite reasonable to expect that the compresison of the toroidal magnetic field may be quite a violent and unstable process on small scales. To predict the resultant relaxed field configuration one might attempt to apply Taylor's theory Taylor 1986 for magnetic reconnection in plasmas. While this is beyond the scope of this letter, possible consequences include topological changes such as magnetic field reversals and reconnection. These in turn should have observational as well as dynamical consequences.

Finally we note that while this work is most relevant to strongly cooling flows such as those associated with YSOs (and Planetary Nebulae) it may also be relevant to the more adiabatic flows associated with AGN and Micro-quasars. While the mass and field compression will be limited to a factor of 4 in an adiabatic flow the rarefaction seen in our model will occur even without cooling. Thus, depending on initial conditions, the alternating toroidal/poloidal geometry may occur in all pulsed jets. 


\section{REFERENCES}

Chrysostomou, A., Gledhill, T. M., Mnard, F., Hough, J. H., Tamura, M., Bailey, J., 2000, MNRAS, 312, 103.

Frank A., Lery T., Gardiner, T.,Jones T. W. \& Ryu D. 1998, ApJ, 494, L79.

Frank A., Ryu D., Jones T. W. \& Noriega-Crespo A. 1998, ApJ, 494, L79.

Gardiner T., Frank A., Ryu D., Jones T., 2000, ApJ, 530, 834.

Heyvaerts, J \& Norman, C., 1989, ApJ, 347, 1055.

Heyvaerts J., Norman C.A., 1989, ApJ, 347, 1055

Kudoh T., Matsumoto R., Shibata K., 1998, ApJ, 508, 186

Leahy J.P., 1991, in "Beams and Jets in Astrophysics", ed. P. Hughes, (Cambridge U. Press).

Lery T., Heyvaerts J., Appl S., Norman C.A., 1998, A\&A 337, 603.

Lery T., Heyvaerts J., Appl S., Norman C.A., 1999, A\&A, in press

Minchin, N. R., Sandell, G., Murray, A. G. 1995, A\&A, 293, 61.

Ouyed R. \& Pudritz R. E. 1997a, ApJ, 482, 712

Pudritz R.E. 1991, in "The Physics of Star Formation and Early Stellar Evolution", eds. C.J. Lada and N.D. Kylafis, NATO ASI Series (Kluwer), 365

Pudritz R., \& Konigl, A., 2000, in Protostars \& Planets IV.

Raga, A. C. \& Kofman, L. 1992, ApJ, 386, 222.

Romanova M. M., Ustyugova, G. V., Koldoba A.V., Chechetkin V.M., \& Lovelace R.V.E. 1998, ApJ, 500, 703

Ray T.P., Mundt R., Dyson J.E., Falle S.A.E.G., Raga A.C., 1996, ApJ, 468, L103.

Reipurth B., 1997, in Herbig-Haro Flows and the Birth of Low Mass Stars, in IAU Symposium no. 182, eds B. Reipurth \& C Bertout (Kluwer, Dordrecht)

Soker N., \& Livio M., 1994, AJ 421, 219.

Spruit, H.C., 2000, in IAU Symposium 195 ed P. Martens, astro-ph/0003043.

Shu F., Najita J., Ostriker E., Wilkin F., Ruden S., \& Lizano S., 1994, ApJ, 429, 781

Smith, M. D., Suttner, G., \& Zinnecker, H. 1997, A\&A, 320, 325. 
Stone, J., Xu, J., Hardee, P., 1997, ApJ, 483, 136.

Taylor, J. B., 1986, Rev. Mod. Phys., 58, 741.

Zinnecker, H., McCaughrean, M. J., Rayner, J. T., 1998, Nature, 394, 862. 
Fig. 1a.- Logarithm of the density after the jet has propagated for 365 years. The colorbar indicates the number density in units of Hydrogen atoms per $\mathrm{cm}^{3}$.

Fig. 1b.- Composite image showing the poloidal magnetic field lines on the top panel and the strength of the toroidal magnetic field component on the bottom panel after propagating for 365 years. The colorbar indicates the toroidal field strength in Gauss.

Fig. 2.- Logarithm of $B_{\phi} / B_{\phi, o}$ along the jet at $1 / 2$ of the jet radius. The two evolutionary times chosen differ by $1 / 2$ of the pulsation period. 